\title{
Expanded Access Study
}

National Cancer Institute

\section{Source}

National Cancer Institute. Expanded Access Study. NCI Thesaurus. Code C98722.

Studies that provide a means for obtaining an experimental drug or device for patients who are not adequately treated by existing therapy, who do not meet the eligibility criteria for enrollment, or who are otherwise unable to participate in another clinical study. Expanded Access studies include individual-patient IND, treatment IND, compassionate use, emergency use or continued access. 